\title{
Les écoulements gaz-liquide en génie chimique
}

\author{
Gas-liquid flows in chemical engineering
}

\author{
Jean-Claude Charpentier \\ Laboratoire des Sciences du Génie Chimique \\ CNRS - ENSIC - Nancy
}

Les écoulements gaz-liquide sont utilisés de façon intensive dans les procédés chimiques, pétrochimiques, pharmaceutiques, parachimiques et dans le cadre de la dépollution des eaux résiduaires et de l'atmosphère. Citons le lavage par des solutions aqueuses des gaz acides $\left(\mathrm{CO}_{2}, \mathrm{H}_{2} \mathrm{~S}, \mathrm{SO}_{2}, \mathrm{SO}_{3}, \mathrm{NO}_{2}, \mathrm{HF}, \mathrm{HCl}, \mathrm{P}_{2} \mathrm{O}_{5} \ldots\right)$, les grandes synthèses chimiques et pétroléochimiques en milieu liquide organique (oxydations, chlorations, hydrogénations, nitrations, sulfonation, polymerisations...), la préparation des produits purs par absorption de solutés gazeux plus ou moins dilués (production de $\mathrm{H}_{2} \mathrm{SO}_{4}, \mathrm{HNO}_{3}, \mathrm{HCl}, \mathrm{H}_{3} \mathrm{PO}_{4}$, des engrais, nitrates, phosphates...), les fermentations et les oxydations biologiques et enfin les transports de mélanges diphasés dans des tuyaux ou des tubes en génies pétrolier et nucléaire.

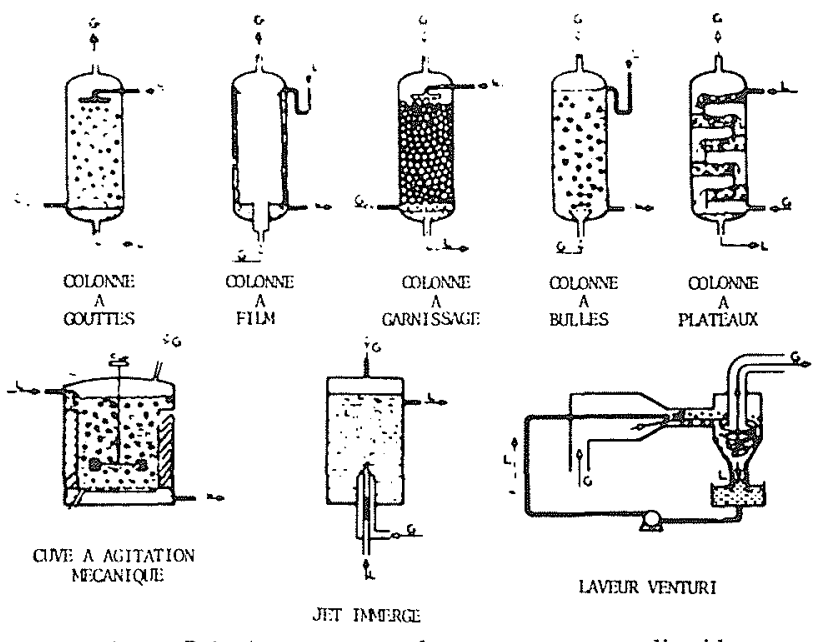

Figure 1. - Principaux types de contacteurs gaz-liquide pour absorption-réaction
Les contacteurs gaz-liquide utilisés ont des formes très diverses telles que les réacteurs tubulaires à gouttes, à film tombant, à bulles, à garnissage à plateaux, les réacteurs à cuve agitée mécaniquement, les réacteurs de type jets ou venturis (fig. 1). Ces différentes formes sont dues à la compétition entre les divers phénomènes de thermodynamique chimique et de cinétiques physiques et chimiques qui interviennent simultanément et qui déterminent le choix de l'appareil devant fonctionner dans des conditions hydrodynamiques et énergétiques optimales.

Voyons maintenant les problèmes spécifiques aux écoulements gaz-liquide qui relèvent des sciences du Génie Chimique.

L'hydrodynamique, la cinétique physique et la cinétique chimique sont intimement imbriquées dans les écoulements diphasiques au sein des réacteurs gaz-liquide

En effet, plaçons-nous dans le cas d'un écoulement gaz-liquide au sein d'un réacteur tubulaire à bulle, de section $\Omega$, qui est le siège d'une réaction irréversible entre un soluté $A$ dissous dans la phase gazeuse puis absorbé par la phase liquide contenant un réactif $B$. La réaction conduit à un produit $P r$ :

$$
A+z B \stackrel{k_{m n}}{\longrightarrow} P r
$$

(par exemple une oxydation avec l'oxygène de l'air en milieu organique). Si l'on suppose que la phase gazeuse, dont le taux de rétention est $\alpha$, est en écoulement piston et que la phase liquide dont le taux de rétention est $\beta=1-\alpha$, est en écoulement piston diffusionnel $\left(D_{L}\right.$ étant le coefficient de dispersion axiale) un bilan de matière et de chaleur dans un petit élément de 


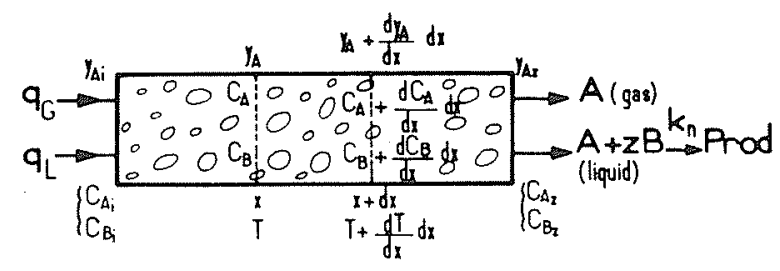

Figure 2.-.- Réacteurs tubulaires

volume $\Omega d x$ (fig. 2) conduit aux équations suivantes en régime permanent:

$$
\begin{aligned}
& -\frac{q_{G}}{\Omega} \frac{P}{R T} \frac{d y_{A}}{d x}-k_{G} a P\left(y_{A}-y_{A}^{*}\right)= \\
& \text { (convection) (transfert entre } \\
& =-\frac{q_{G}}{\Omega} \frac{P}{R T} \frac{d y_{A}}{d x}-E k_{L} a\left(C_{A}^{*}-C_{A}\right)=0 \\
& (1-\alpha) D_{L} \frac{d^{2} C_{A}}{d x^{2}}-\frac{q_{L}}{\Omega} \frac{d C_{A}}{d x}+ \\
& \text { (dispersion (convec- } \\
& \text { axiale) tion) } \\
& +E k_{L} a\left(C_{A}^{*}-C_{A}\right)+(1-\alpha) r_{A}=0 \\
& \text { (transfert entre (réaction } \\
& \text { phases) } \\
& \text { chimique) } \\
& (1 \cdots \alpha) D_{L} \frac{d^{2} C_{B}}{d x^{2}}-\frac{q_{L}}{\Omega} \frac{d C_{B}}{d x}+(1-\alpha) z r_{A}=0 \\
& \text { (dispersion (convec- (réaction } \\
& \text { axiale) tion) chimique) }
\end{aligned}
$$

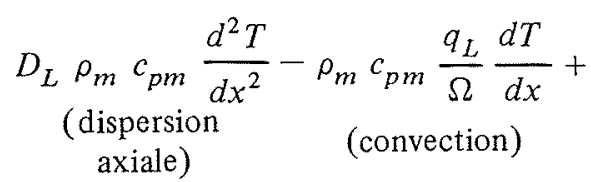$$
\begin{array}{cc}
(1-\alpha) r_{A} \Delta H_{A} & -\mathcal{U} \mathcal{Q}\left(T-T_{e}\right)=0 \\
\begin{array}{c}
\text { (réaction } \\
\text { chimique) }
\end{array} & \text { (conductance vers } \\
\text { l'extérieur) }
\end{array}
$$

La première équation signifie que pendant le déplacement par convection du gaz la diminution $d y_{A}$ du titre en soluté en phase gazeuse est due au flux $\Phi$ de matière transféré à la phase liquide au travers de l'aire spécifique d'interface $a$ par l'intermédiaire des conductances partielles de transfert en phase gazeuse $k_{G}$ et en phase liquide $k_{L}$ qui permettent au corps $A$ de diffuser de chaque côté de l'interface sous l'influence d'une différence de potentiel de transfert $\left(p-p_{i}\right)$ ou $\left(C_{A}^{*}-C_{A}\right)$. Dans cette expression du flux, on modélise la "cinétique physique" du transfert par le modèle du double film (fig. 3). $E$ est le facteur d'accélération due à la réaction chimique qui caractérise l'effet de la présence de la réaction chimique sur la cinétique physique du transfert de matière ( $E=1$ pour une absorption en absence de réaction chimique).
La deuxième équation signifie que pendant le mouvement par dispersion axiale et convection du liquide, le soluté transféré de la phase gazeuse et donc dissous réagit avec le réactif $B$ avec une vitesse de réaction $r_{A}$. C'est la "cinétique chimique". $r_{A}$ est fonction des concentrations des réactifs au sein du liquide, par exemple, (au sein du liquide où la concentration en gaz dissout est $\left.C_{A}\right)$ :

$$
r_{A}=-\frac{d C_{A}}{d t}=k_{A B} C_{A} C_{B}
$$

si la réaction est d'ordre partiel 1 pour chacun des réactifs en présence, $k_{A B}=k_{m n}=k_{2}$ étant la constante cinétique de la réaction.

La troisième équation relative au réactif $B$ contient les mêmes termes que la précédente à l'exception du terme de transfert si l'on suppose que le réactif $B$ n'est pas volatil.

La quatrième équation concerne le transfert de chaleur au sein du mélange de densité $\rho_{m}$ et de capacité calorifique $c_{p m}$. Pendant le mouvement d'ensemble par convection et dispersion axiale, un flux de chaleur est créé ou demandé par la réaction chimique $\left(\Delta H_{A}\right.$ est l'enthalpie de la réaction) et un flux de chaleur évacué ou apporté par l'extérieur où la température est $T_{e}$ par conductibilité thermique (cas d'un serpentin de chauffage ou de refroidissement). $\mathcal{Q}$ est la surface spécifique d'échange thermique et $\mathcal{U}$ le coefficient global de transfert de chaleur.

Les équations (1) à (4) montrent à quel point les phénomènes concernant l'hydrodynamique des écoulements (caractérisé par le taux de rétention de chaque

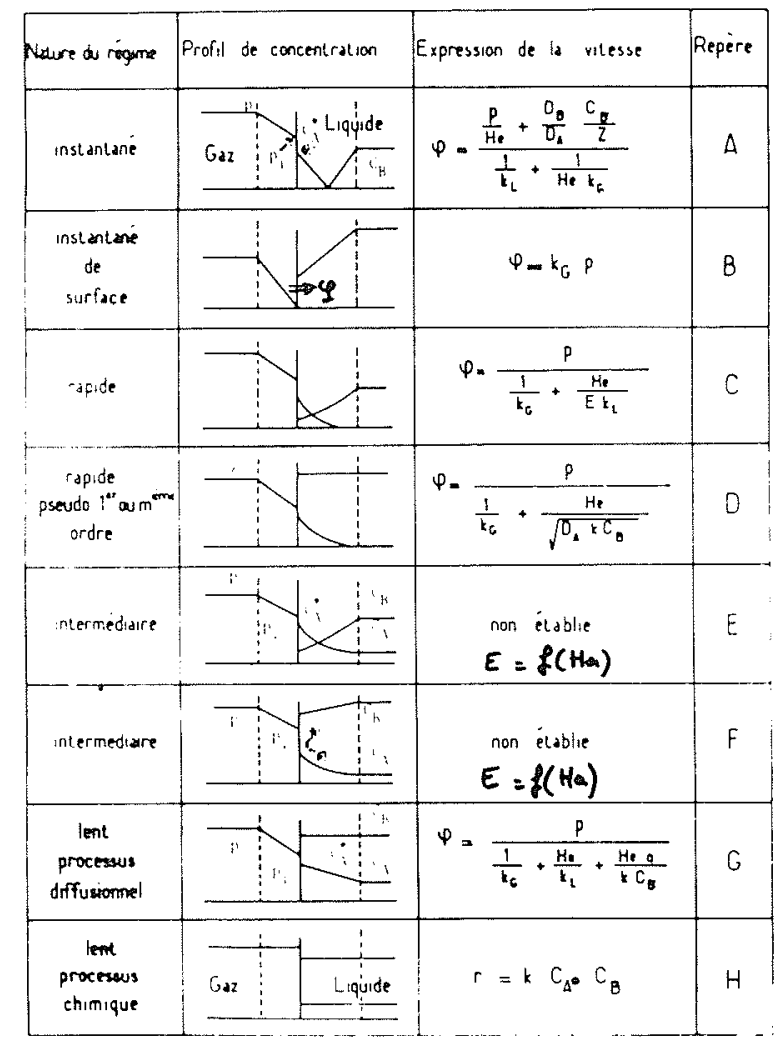

Figure 3 - Les différents régimes réactionnels de l'absorption gaz-liquide avec réaction chimique [3]. 
phase et éventuellement la dispersion axiale dans chaque phase), la thermodynamique chimique (solubilité $C_{A}^{*}=y_{A}^{*} P / H e=p_{i} / H e$ de soluté dissous, diffusivités $D_{A}, D_{B}$ des réactifs dans le liquide, l'enthalpie de la réaction $\Delta H_{A}$ ), la cinétique physique caractérisant le transfert de matière et/ou de chaleur $\left(k_{G} a, a, k_{L} a, U \ldots\right)$ et la cinétique chimique (constante cinétique $k_{n}$ et ordre de la réaction) sont étroitement imbriquées. En effet c'est seulement quand on connait les valeurs expérimentales des différents paramètres précédents caractéristiques des écoulements diphasiques, que l'on peut résoudre les équations et disposer des profils de température $T(x)$ et/ou de concentration

$$
y_{A}(x)=\frac{p_{A}(x)}{P}, C_{A}(x), C_{B}(x)
$$

en fonction de la longueur du contacteur. On voit ainsi dans notre exemple que le choix puis le calcul de la longueur d'un réacteur tubulaire $Z$, pour un taux de conversion en soluté $A, X_{A}=\left(y_{A i}-y_{A z}\right) / y_{A i}$ imposé, nécessite la connaissance d'un grand nombre de paramètres diphasiques et principalement ceux caractérisant ou intervenant dans le flux global de transfert de matière entre phases,

$$
\begin{aligned}
\Phi=\phi \cdot a=k_{G} a P\left(y_{A}-y_{A}^{*}\right) & = \\
=k_{G} a\left(p-p_{i}\right) & =E k_{L} a\left(C_{A}^{*}-C_{A}\right)
\end{aligned}
$$

et leur évolution lors d'une extrapolation à plus grande échelle pour un contacteur de forme donnée ou pour différentes formes d'appareils. En effet c'est principalement la compétition qui existe entre la cinétique physique et la cinétique chimique au niveau du transfert de matière entre phases qui conditionne le choix de l'appareil puis son dimensionnement. Cela apparaît clairement sur la figure 3 [3] qui représente les différents profils de concentrations des réactifs $A$ et $B$ de chaque côté de l'interface, d'abord au sein de chaque phase où la concentration est uniforme puis dans les films laminaires d'épaisseur $\delta_{G}=D_{G} / k_{G}$ et $\delta_{L}=D_{A} / k_{L}$ $\left(D_{G}\right.$ est la diffusivité du soluté $A$ dans le mélange gazeux, $D_{G}=0$ pour un gaz pur) où se localisent la résistance au transfert de masse et/ou la réaction chimique. D'après l'expression du taux d'absorption $\phi$ on constate que pour les rćactions instantanćcs ou rapides (cas $A, B, C, D$ de la figure 3 ) toute la réaction se tient dans le film côté liquide $C_{A}=0$ et non pas au sein $d u$ liquide (il vient $r_{A}=0$ dans l'équation 2). Donc les paramètres principaux qui augmentent le flux de transfert $\Phi=\phi . a$ sont les conductances de transfert $k_{L}$ et $k_{G}$ et l'aire spécifique interfaciale $a$. C'est le "régime de cinétique physique", caractérisé en général par le critère $H a=\sqrt{D_{A} k_{2} C_{B}} / k_{L}>3$. Pour ce type de réaction en écoulement diphasique les contacteurs utilisés ne nécessitent pas un taux de rétention de liquide $\beta=1-\alpha$ important : colonnes à gouttes, à garnissage ou réacteurs de type jets ou venturis.

A l'opposé, pour les procédés chimiques lents (cas $H$ de la figure 3), le flux de transfert où la cinétique physique n'intervient plus $\left(C_{A}^{*}=C_{A}\right)$, c'est la réaction chimique au sein du liquide qui limite le procédé. Il ne sert à rien de dépenser de l'énergie pour créer une

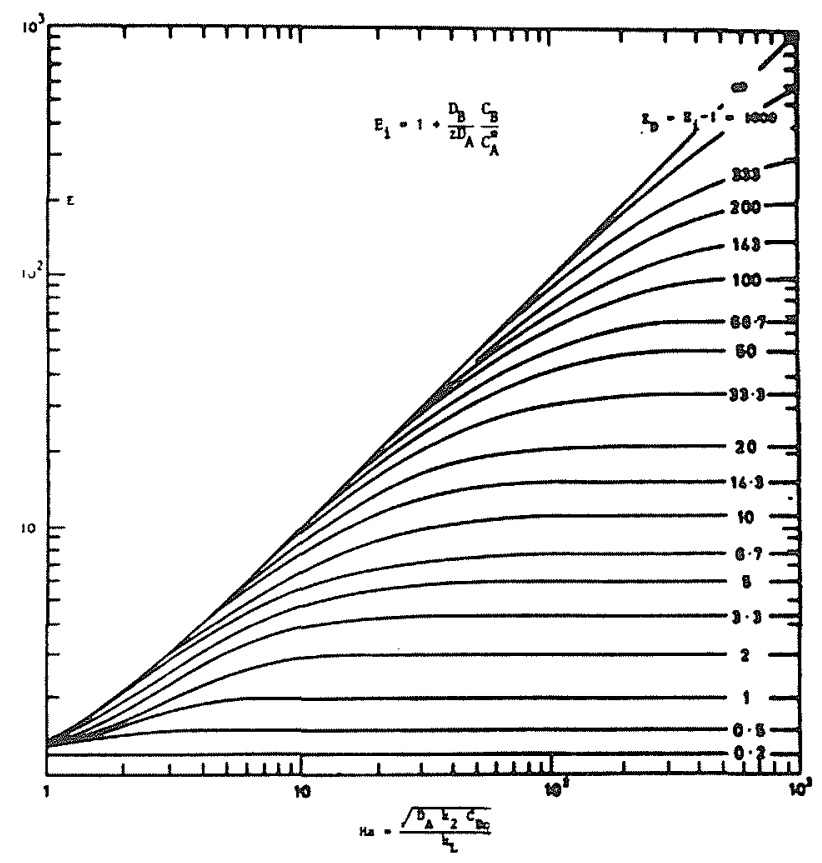

Figure 4 - Variations du facteur d'accélération en fonction du critère de Hatta

augmentation de $k_{L}, k_{G}$ ou $a$. On a besoin au contraire d'un taux de rétention de liquide important et d'une grande teneur en catalyseur pour augmenter la vitesse de réaction. C'est le "régime de cinétique chimique" caractérisé par $H a \ll 1$. Une colonne à bulle conviendra parfaitement.

Pour les régimes intermédiaires (cas $E, F, G$ de la figure 3) où la réaction peut se tenir à la fois dans le film liquide au voisinage de l'interface et au sein du liquide, les cinétiques physique et chimique ont un poids comparable dans le processus global de transfert de matière et il faudra simultanément des valeurs importantes de $k_{L}, k_{G}$, a et $\beta$. C'est le cas des colonnes à plateaux, des réacteurs à cuve agitée mécaniquement et des jets immergés. Le flux spécifique de transfert peut avoir une expression explicite (cas $G$ ) ou ne pas en avoir. Dans ce dernier cas, on le calculera par l'équation (5) où les valeurs du facteur d'accélération $E$ en fonction du critère de $H a$ sont représentées graphiquement sur la figure 4 proposée pour une réaction irréversible d'ordre global 2 mais en fait valable pour des ordres plus élevés [9]. Le critère $H a$ ou paramètre de diffusion-réaction est un paramètre important dont la signification physique apparait clairement dans les expressions suivantes:

conversion maximum dans le film d'épaisseur $\delta_{L}$

flux de matière maximum transporté

par diffusion à travers ce film

$$
=\frac{k_{2} C_{A}^{*} C_{B} \delta_{L}}{D_{A}\left|\frac{C_{A}^{*}-0}{\delta_{L}}\right|}=\frac{k_{2} C_{B} \delta_{L}^{2}}{D_{A}}=\frac{D_{A} k_{2} C_{B}}{k_{L}^{2}}=H a^{2}
$$


ou encore

$$
\frac{\text { temps de diffusion }}{\text { temps de réaction }}=\frac{t_{D}}{t_{r}}=\frac{\frac{D_{A}}{k_{L}^{2}}}{\frac{1}{k_{2} C_{B}}}=\frac{D_{A} k_{2} C_{B}}{k_{L}^{2}}=H a^{2}
$$

Le calcul du critère Ha pour un système réactionnel diphasique donné permet donc de déterminer le type de réacteur à choisir à condition bien sûr de connaître les paramètres de cinétiques chimique et physique qui interviennent dans son expression.

La modélisation se fait ensuite par l'intermédiaire des équations (1) à (5) dans le cas d'écoulements diphasiques dans des contacteurs tubulaires ou des équations équivalentes pour des contacteurs de type cuve agitée où la phase gazeuse et/ou la phase liquide sont parfaitement agitées $[1,9]$

Pour résoudre les équations, afin de dimensionner en longueur ou en volume le contacteur, il faut donc connaitre les valeurs expérimentales des paramètres caractérisant le transfert, la réaction chimique et l'hydrodynamique puis la variation de ces paramètres avec les débits des phases et/ou l'énergie fournie au mélange diphasique ce qui sera utile lors de l'extrapolation à plus grande échelle. L'hydrodynamique intervient encore au niveau du dimensionnement en diamètre car ce dernier est tributaire des régimes d'écoulement gazliquide, des phénomènes d'engorgement ou des problèmes de moussage ... ce qui montre, et j'insiste volontairement, qu'il ne faut jamais dissocier les différents phénomènes d'hydrodynamique et de cinétique plyysique et chimique dans les systèmes diphasiques rencontrés couramment dans les écoulements gaz-liquide en Génie Chimique.

Avant d'utiliser un contacteur de forme classique comme ceux de la figure 1 mais en régime de fonctionnement non classique ou bien un contacteur de conception nouvelle, on conçoit maintenant qu'il faut faire une étude fournissant les paramètres hydrodynamiques et ceux du transfert de matière à savoir le taux de rétention de chaque phase, l'aire interfaciale, les conductances de transfert et ce pour les différents régimes d'écoulement rencontrés. Je ne rappellerai ici que le principe de la méthode chimique de mesure des grandeurs caractérisant le transfert de masse puisque c'est cette méthode qui a été utilisée dans chacun des exemples présentés dans les conférences suivantes : colonne à bulles fonctionnant jusqu'à l'envolement du liquide, contacteur gaz-liquide de type multicellulaire et laveur de type jet pulvérisé de liquide dans un venturi

Méthode chimique de mesure des aires interfaciales et des coefficients de transfert de matière : méthode simple mais non exhaustive

Le principe de la méthode est d'effectuer une absorption gaz-liquide accompagnée d'une réaction chimique. Nous venons de voir qu'il existe une compétition entre le transfert de matière et la réaction chimique. Suivant que c'est l'un ou l'autre de ces phénomènes qui est le plus lent on peut avoir accès soit à $k_{G} a, a$ ou $k_{L} a$ [7].

En effet, reportons-nous à nouveau à la figure 3 . Le régime de réaction instantanée et de surface (cas $B$ ) conduit à :

$$
\Phi=\phi \cdot a=k_{G} a \cdot p=k_{G} a \cdot y_{A} \cdot P
$$

la condition à remplir pour avoir un tel régime en tout point du réacteur est :

$$
k_{G} a . p<k_{L} a \frac{D_{B}}{z D_{A}} \frac{C_{B}}{C_{A}^{*}}
$$

L'intégration de (6) sur toute la longueur $Z$ du réacteur conduit à :

$$
k_{G} a=\frac{G}{P Z \Omega} \log \frac{y_{A i}}{y_{A Z}}
$$

Une mesure des titres à l'entrée $y_{A i}$ et à la sortie $y_{A z}$ du soluté gazeux fournit la valeur globale de $k_{G} a$ ( $G$ est le débit molaire de la phase gazeuse). La réaction entre $\mathrm{SO}_{2}$ dilué dans l'air et une solution aqueuse de soude ou de carbonate de sodium est généralement employée.

Par ailleurs le régime de réaction rapide de pseudo premier ou pseudo $\mathrm{m}^{\mathrm{e}}$ ordre en soluté gazeux (cas $D$ ) avec de plus une absence de résistance en phase gazeuse $\left(1 / k_{G}=0\right)$ conduit à :

$$
\Phi=\phi . a=a \cdot \frac{p_{i}}{H e} \sqrt{D_{A} k_{2} C_{B}}=a \cdot C_{A}^{*} \sqrt{D_{A} k_{2} C_{B}}
$$

pour une réaction de pseudo premier ordre et à :

$$
\Phi=\phi . a=a \cdot \sqrt{\frac{2}{m+1} k_{m n} D_{A} k_{m n} C_{A}^{* m+1} C_{B}^{n}}
$$

pour une réaction de pseudo $\mathrm{m}^{\mathrm{e}}$ ordre.

La condition à remplir est $3<H a \ll E_{i}$, soit encore

ou

$$
3<\frac{\sqrt{D_{A} k_{2} C_{B}}}{k_{L}}<1+\frac{D_{B}}{z D_{A}} \frac{C_{B}}{C_{A}^{*}}
$$

$3<\frac{1}{k_{L}} \sqrt{\frac{2}{m+1} D_{A} k_{m n} C_{A}^{* m-1} C_{B}^{n}}<1+\frac{D_{B}}{z D_{A}} \frac{C_{B}}{C_{A}^{*}}$

Si cette condition est remplie en tout point du réacteur, la mesure de la quantité de soluté absorbée entre l'entrée et la sortie fournit l'aire interfaciale par l'équation (7) quand on connait $C_{A}^{*} \sqrt{D_{A} k_{2} C_{B}}$.

Les réactions entre $\mathrm{CO}_{2}$ dilué dans l'air et une solution aqueuse de soude et entre l'oxygène de l'air et une solution de sulfite de sodium catalysée par des ions cobalt $\left(k_{m n} C_{B}^{n}=k_{c}, C_{0}^{++}\right)$sont généralement utilisées. Pour des concentrations en réactif remplissant les conditions $7 \mathrm{a}$ et $7 \mathrm{~b}$, les ordres partiels en soluté gazeux sont respectivement $m=1$ et $m=2$.

Enfin le régime de réaction chimique lente donne accès aux valeurs de $k_{L}$ a (fig. 3 , cas $G$ ). Un tel régime 


\begin{tabular}{|c|c|c|c|c|}
\hline \multirow[b]{2}{*}{ Type de réacteur } & \multicolumn{4}{|c|}{ Paramètres caractéristiques des différents types de réacteurs gaz-liquide [9] } \\
\hline & $\begin{array}{c}\beta \\
(\%)\end{array}$ & $\left(\mathrm{mole} / \mathrm{cm}^{2} \cdot{ }^{k}\right.$. atm $) 10^{4}$ & $\begin{array}{c}k L \\
(\mathrm{~cm} / \mathrm{s}) \cdot 10^{2}\end{array}$ & $\mathrm{~cm}^{2} / \mathrm{cm}^{3}$ réacteur \\
\hline $\begin{array}{l}\text { Colonnes à garnissage } \\
- \text { contre-courant } \\
\text { - co-courant }\end{array}$ & $\begin{array}{l}2-25 \\
2-95\end{array}$ & $\begin{array}{l}0,03-2 \\
0,1-3\end{array}$ & $\begin{array}{ll}0,4 & -2 \\
0,4 & -6\end{array}$ & $\begin{array}{l}0,1-3,5 \\
0,1-17\end{array}$ \\
\hline $\begin{array}{l}\text { Colonnes à plateaux } \\
\text { - calotes } \\
\text { - perforées }\end{array}$ & $\begin{array}{l}10-95 \\
10-95\end{array}$ & $\begin{array}{ll}0,5 & -2 \\
0,5 & -6\end{array}$ & $\begin{array}{ll}1 & -5 \\
1 & -20\end{array}$ & $\begin{array}{ll}1 & -4 \\
1 & -2\end{array}$ \\
\hline$\left\{\begin{array}{l}\text { Colonnes à bulles } \\
\text { Colonnes garnies à bulles }\end{array}\right.$ & $\begin{array}{l}60-98 \\
60-98\end{array}$ & $\begin{array}{ll}0,5 & -2 \\
0,5 & -2\end{array}$ & $\begin{array}{ll}1 & -4 \\
1 & -4\end{array}$ & $\begin{array}{l}0,5-6 \\
0,5-3\end{array}$ \\
\hline $\begin{array}{l}\text { Réacteurs tubulaires } \\
\text { - horizontaux et à serpentins } \\
\text { - verticaux }\end{array}$ & $\begin{array}{l}5-95 \\
5-95\end{array}$ & $\begin{array}{ll}0,5 & 4 \\
0,5 & -8\end{array}$ & $\begin{array}{ll}1 & -10 \\
2 & -5\end{array}$ & $\begin{array}{l}0,7-7 \\
1-10\end{array}$ \\
\hline Colonnes à gouttes & $2-20$ & $0,5-2$ & $0,7-1,5$ & $0,1-1$ \\
\hline Réacteurs à cuves agitées mécaniquement & $20-95$ & - & $0,3-7$ & $1-10$ \\
\hline $\begin{array}{l}\text { Jets plongeants et immergés } \\
\text { - hydrocyclone } \\
\text { - réacteur ejecteur } \\
\text { - venturi }\end{array}$ & $\begin{array}{c}94-99 \\
70-93 \\
- \\
5-30\end{array}$ & $\begin{array}{l}- \\
- \\
2-10\end{array}$ & $\begin{array}{c}0,15-0,5 \\
10-30 \\
- \\
5-10\end{array}$ & $\begin{array}{c}0,2-1,2 \\
0,2-0,5 \\
1-20 \\
1,6-25\end{array}$ \\
\hline
\end{tabular}

est obtenu quand il n'y a pas de réaction chimique dans le film liquide au voisinage de l'interface $(E=1)$, mais au sein du liquide la réaction est toutefois suffisamment rapide pour que le réactif $A$ soit consommé $\left(C_{A}=0\right)$. L'expression (5) du flux s'écrit alors :

$$
\Phi=\phi \cdot a=k_{L} a \cdot C_{A}^{*}
$$

et la mesure de la quantité de soluté absorbée entre l'entrée et la sortie fournit $k_{L} a$ quand on connait la solubilité $C_{A}^{*}$. On suppose également une absence de résistance en phase gazeuse.

Les conditions à remplir en tout point du réacteur sont :

$$
H a<1 \text { et } k_{L} a \ll \beta k_{m n} C_{A}^{* m-1} C_{B}^{n}
$$

Les systèmes chimiques employés sont à nouveau l'oxydation des sulfites catalysés par des ions cobalt et l'absorption de $\mathrm{CO}_{2}$ dissous dans l'air par des solutions tampons de carbonate et bicarbonate de soude.

L'avantage apparent de la méthode chimique est de fournir des valeurs globales des paramètres physiques $a, k_{L} a$ et $k_{G} a$ par rapport aux valeurs locales obtenues par les méthodes purement physiques de réflexion ou d'absorption de la lumière ou photographique. En réalité ces deux méthodes sont complémentaires et doivent ètre utilisées conjointement et complémentairement toutes les fois que ce sera possible.

On trouvera dans le tableau $1[5,6]$ un ordre de grandeur de ces paramètres dans les différents types classiques de réacteurs gaz-liquide rencontrés couramment dans la pratique industrielle.

Et s'il n'est pas possible d'évaluer expérimentalement ou de trouver dans la littérature les valeurs des différents paramètres diphasiques, comment faire pour extrapoler?

\section{La notion de simulation sur maquettes de laboratoire}

Dans les paragraphes précédents, nous avons admis que la solubilité $C_{A}^{*}$, les diffusivités $D_{A}$ et $D_{B}$ des réac- tifs et la cinétique chimique étaient parfaitement connues ce qui permet d'évaluer le flux de transfert entre phases. Dans le cas de réactions chimiques réversibles, consécutives, parallèles, ou dans le cas d'absorption simultanée de plusieurs solutés suivie de réactions plus ou moins complexes en phase liquide, la recherche de la valeur des paramètres caractéristiques de la thermodynamique et de la cinétique chimique peut être un problème inextricable. Dans ces conditions, pour l'extrapolation à l'échelle pilote et/ou à l'échelle industrielle, il n'est plus question d'utiliser les solutions des équations (1) à (5). Il est également très onéreux de mener une série systématique d'expériences sur des équipements dont les dimensions vont en croissant. Ces difficultés ont conduit à imaginer et construire des maquettes de laboratoire ayant une aire interfaciale et des valeurs de $k_{L}$ et $k_{G}$ connues ou très simplement mesurables (fig. 5) et dans lesquelles il serait possible de pré-

\begin{tabular}{|c|c|c|c|c|c|c|c|c|}
\hline \multirow{2}{*}{ MODEL } & \multirow{2}{*}{$\begin{array}{l}\text { ROTA } \\
\text { IING } \\
\text { DRUM }\end{array}$} & \multirow{2}{*}{$\begin{array}{l}\text { MOVING } \\
\text { BAND }\end{array}$} & \multirow{2}{*}{$\begin{array}{l}\text { LAMI } \\
\text { NAR } \\
\text { JET }\end{array}$} & \multicolumn{3}{|c|}{ WETTED WALL COLUMN } & \multirow{2}{*}{$\begin{array}{l}\text { STRING } \\
\text { OF } \\
\text { OISCS. } \\
\text { SPEERES }\end{array}$} & \multirow{2}{*}{$\begin{array}{l}\text { STIRRED } \\
\text { VESSE: }\end{array}$} \\
\hline & & & & SPHERE & CYLIN & CONE & & \\
\hline $\begin{array}{l}\text { TIME OF } \\
\text { CONIACT } \\
(s)\end{array}$ & $\begin{array}{l}2.10^{-4} \\
10^{-1}\end{array}$ & $\begin{array}{l}0.10^{-6} \\
6.10^{-2}\end{array}$ & $\begin{array}{l}10^{-3} \\
10^{-1}\end{array}$ & $\begin{array}{l}10^{-1} \\
1\end{array}$ & $\begin{array}{c}10^{1} \\
2\end{array}$ & $\begin{array}{c}\therefore .10^{-1} \\
1\end{array}$ & $\begin{array}{l}10^{-1} \\
2\end{array}$ & $\begin{array}{c}6.10^{\prime 2} \\
10\end{array}$ \\
\hline $\begin{array}{l}\text { NIERFACIAL } \\
\text { AREA } \\
A_{\mathrm{m}}\left(\mathrm{cm}^{2}\right)\end{array}$ & $\begin{array}{c}2 \\
100\end{array}$ & $\begin{array}{c}0.8 \\
0\end{array}$ & $\begin{array}{l}0.3 \\
10\end{array}$ & $\begin{array}{l}10 \\
40\end{array}$ & $\begin{array}{l}10 \\
100\end{array}$ & 00 & $\begin{array}{l}30 \\
300\end{array}$ & i. \\
\hline$k_{L}\left(\varepsilon m, s^{-1}\right)$ & $\begin{array}{l}0.010 \\
0.358\end{array}$ & $\begin{array}{l}0.021 \\
0.210\end{array}$ & $\begin{array}{l}0.010 \\
0.100\end{array}$ & $\begin{array}{l}0.005 \\
0.018\end{array}$ & $\begin{array}{l}0.00301 \\
0.01\end{array}$ & 0.005 & $\begin{array}{l}0.0038 \\
0.010\end{array}$ & $\begin{array}{l}0.0018 \\
0.021\end{array}$ \\
\hline 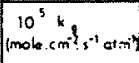 & - & - & $\begin{array}{l}10 \\
40\end{array}$ & & & & : & $\begin{array}{c}3 \\
15\end{array}$ \\
\hline $\begin{array}{l}A_{m} / v_{m} \\
\left(\mathrm{~cm} \cdot{ }^{\prime}\right)\end{array}$ & $\begin{array}{r}100 \\
1250\end{array}$ & 100 & $\begin{array}{l}20 \\
80\end{array}$ & 20 & $\begin{array}{l}25 \\
00\end{array}$ & $\begin{array}{l}10 \\
10\end{array}$ & $\begin{array}{l}20 \\
00\end{array}$ & $\begin{array}{l}0.002 \\
0.540\end{array}$ \\
\hline
\end{tabular}

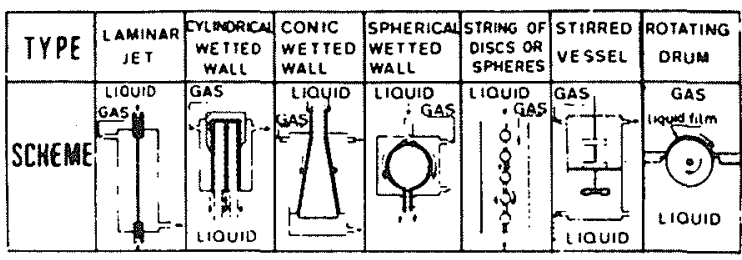

Figure 5 - Principales maquettes de laboratoire [6]. 
dire ou "simuler" le comportement du système diphasique dans l'appareil industriel envisagé ou tout du moins d'obtenir des résultats expérimentaux directement utilisables pour l'extrapolation. Le but recherché est donc de prévoir l'effet de telle ou telle réaction chimique lors d'un écoulement diphasique industriel à partir d'expériences effectuées sur le modèle de labo. ratoire avec le même système gaz-liquide ou bien de dimensionner en longueur un réacteur pour une réaction gaz-liquide imposée, toujours à partir de résultats obtenus avec la maquette, même si la façon de mettre en contact les deux phases est tout à fait différente.

En comparant les équations caractérisant le bilan de matière dans la maquette, et dans l'équipement à simuler, il apparait qu'une telle simulation n'est possible que si certains critères, suivant le type de réaction rencontrée, sont respectés : mêmes valeurs de $k_{L}, k_{G}$ (ce qui conduit à la même valeur du flux spécifique de transfert ou taux d'absorption $\phi), a / \beta$, $q_{L} / q_{G}, q_{L} / S$ (S est ici l'aire interfaciale rapportée à une unité de volume).

Ainsi lorsque les critères précédents ont les mêmes valeurs dans les deux appareils, "simuler" signifie que si la composition au sein du gaz et du liquide est la même dans un élément de volume du réacteur industriel et dans la maquette, les taux d'absorption $\phi=\phi_{m}$ seront identiques, quelle que soit la façon de mettre en contact les deux phases dans les deux équipements. Alors $\phi_{m}$ sera mesuré expérimentalement dans la maquette de laboratoire en fonction des différentes valeurs de $y_{A}, C_{A}$ et $C_{B}$ correspondant à celles des différentes tranches comprises entre l'entrée et la sortie du réacteur à simuler. L'intégration de l'ensemble de ces valeurs permet d'évaluer la longueur du réacteur [3] : c'est la simulation différentielle. Une comparaison du tableau 1 et de la figure 5 montre qu'un film tombant ou un réacteur agité de laboratoire peut simuler une colonne à garnissage (même gamme de valeurs de $k_{L}$ et $k_{G}$ ) pour des réactions se tenant dans le film liquide au voisinage de l'interface.

Pour des réactions plus ou moins complexes se tenant au sein du liquide, nécessitant un taux de rétention du liquide $\beta$ important, l'égalité des critères $a / \beta$ dans les deux appareils est requise. Pour des équipements industriels, qui n'ont pas une forme cylindrique, il n'est plus question de mesurer expérimentalement dans la maquette les taux d'absorption $\phi_{m}$ correspondant aux différentes valeurs des pressions partielles ou concentrations entre l'entrée et la sortie de l'appareil industriel. Dans ces cas, la maquette de laboratoire simulera le réacteur gaz-liquide quand les compositions des phases à l'entrée et à la sortie des deux équipements seront les mêmes sans qu'il soit nécessaire de faire la moindre hypothèse sur le mécanisme du transfert de matière ou sur la cinétique chimique de la réaction. Seuls les critères de simulation doivent être respectés : c'est la simulation intégrale $[6,10]$. Nous en aurons un exemple dans la conférence sur le réacteur jet dans un venturi : un jet laminaire de diamètre $0,7 \mathrm{~mm}$ et de longueur $2 \mathrm{~cm}$ foumira les mêmes valeurs de $y_{A i} / y_{A z}$ qu'un réacteur jet turbulent dans un venturi de longueur environ $1 \mathrm{~m}$ avec une buse d'éjecteur de $4 \mathrm{~mm}$ et cela avec des rapports des débits des phases liquides et gazeuses dans chaque appareil respectivement d'environ $q_{L} / q_{L m}=300$ et $q_{G} / q_{G m}=30000$.

Cette technique de simulation est également particulièrement intéressante et prometteuse quand, dans un réacteur gaz-liquide existant, il est nécessaire de changer la nature d'une ou des deux phases (par exemple le remplacement de liquides aqueux par des liquides organiques). La prédiction des nouvelles performances peut être alors obtenue à partir d'expériences menées sur la maquette de laboratoire avec le nouveau système gazliquide en respectant les critères de simulation [11].

On voit donc une fois de plus que pour utiliser les écoulements gaz-liquides dans les réacteurs ou absorbeurs, il est nécessaire de connaître $a, k_{L}$ et/ou $k_{G}$, quelle que soit la technique utilisée pour le dimensionnement en longueur ou en volume.

Dans l'emploi de la méthode chimique (voir plus haut) ces coefficients peuvent être mesurés si l'on connait la solubilité, la diffusivité et la cinétique du système réactionnel utilisé. Inversement les paramètres de thermodynamique et cinétique chimiques peuvent être déterminés expérimentalement si on connait les paramètres de la cinétique physique. C'est le cas dans les maquettes de laboratoire de la figure 5 et c'est la raison pour laquelle ces maquettes sont également utilisées pour étudier les cinétiques chimiques des réactions qui seront ensuite mises en œuvre pour évaluer dans les installations pilotes ou dans les réacteurs industriels les valeurs de $a$, $k_{L} a$ et $/$ ou $k_{G} a$.

\section{Conclusions : l'utilisation des écoulements gaz- liquide dans des réacteurs couvrant des besoins très différents de la pratique industrielle}

Comme le lecteur a pu s'en rendre compte, les écoulements gaz-liquide rencontrés dans les industries chimiques et annexes soulèvent de nombreux problèmes qui sont le plus souvent très complexes. En effet, dans les paragraphes précédents, nous nous sommes volontairement placés dans le cas de réactions chimiques dont la cinétique peut être exprimée simplement. Nous n'avons pas fait intervenir les notions d'équilibre, de sélectivité, l'influence de la température sur les paramètres de la thermodynamique et cinétique chimiques qui peut conduire à des états multiples de fonctionnement (stables ou instables) pour une même hydrodynamique des écoulements [6]... Toutefois ces phénomènes devront être parfaitement dominés avant même la mise en œuvre du système diphasique, que ce soit dans les domaines chimiques, biochimiques, pétrochimiques ou tout autre.

C'est pourquoi la méthodologie, l'ingénierie et la technologie qui président à la conception et au fonctionnement optimal d'un réacteur ou d'un absorbeur gaz-liquide relèvent des sciences du Génie Chimique : modélisation des cinétiques physiques des transferts de matière et de chaleur entre phases, et des cinétiques chimiques, compétition entre ces cinétiques, simulation, optimisations énergétiques et extrapolation. Le lecteur trouvera dans les références $[1-10]$ de précieux renseignements sur les considérations théoriques et les valeurs expérimentales nécessaires à la modélisation 


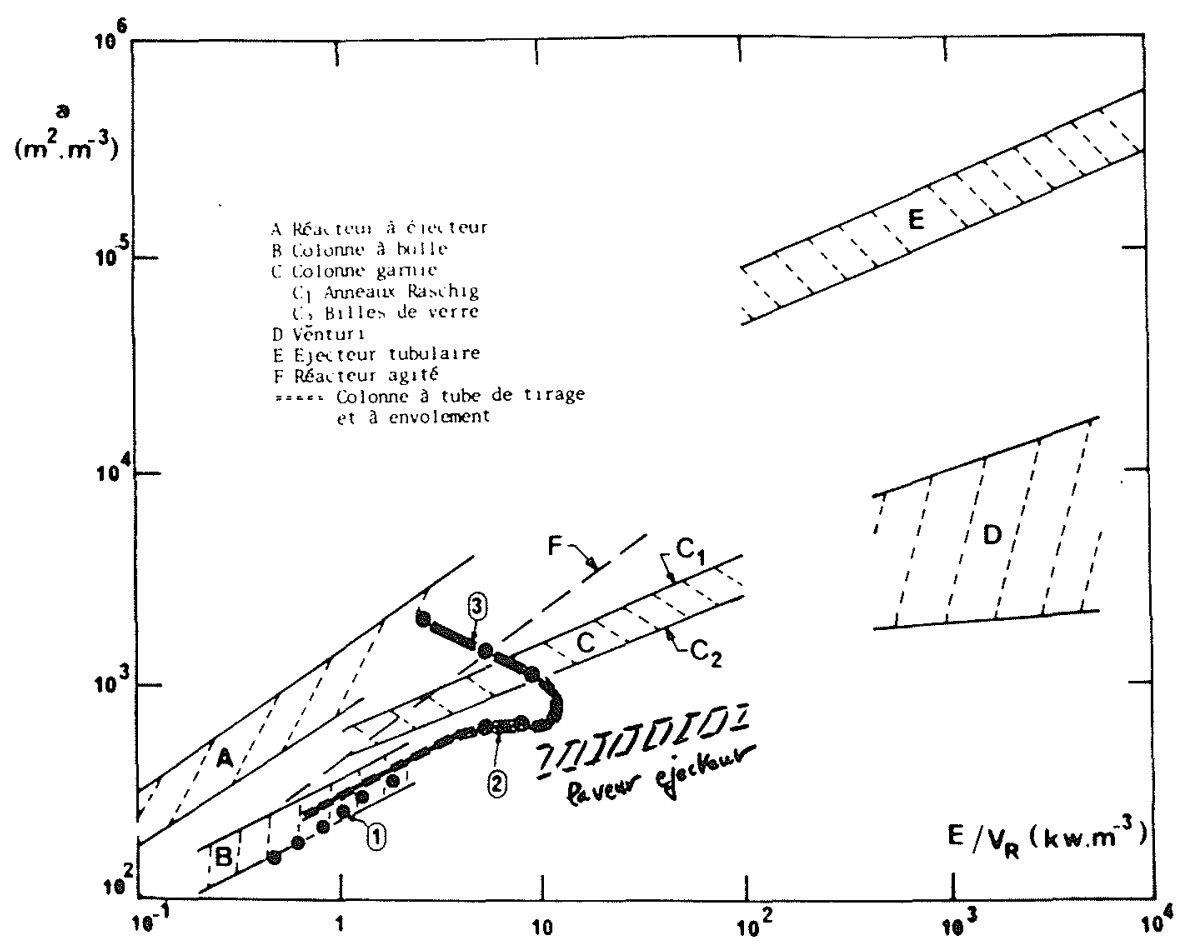

Figure 6 - Aire interfaciale en fonction de la dépense énergétique.

conduisant au choix du réacteur et du type d'écoulement gaz-liquide dans ce réacteur. Pour l'optimisation énergétique et l'extrapolation, les courbes des diagrammes du type de celui de la figure 6 lui permettront de préciser la dépense énergétique pour une aire interfaciale spécifique donnée.

Pour illustrer les considérations précédentes, les résultats des recherches expérimentales et complémentaires sur trois contacteurs gaz-liquide très différents, et pouvant donc satisfaire des besoins très différents, vont être présentés dans les conférences suivantes. Le premier contacteur sera une colonne à bulle dans un état de fonctionnement non classique, soit munie d'un tube de tirage, soit fonctionnant avec un très grand débit de gaz où tous les états hydrodynamiques seront présents (voir également la figure 6). Le deuxième contacteur sera un absorbeur de type multicellulaire, de forme non classique qui pourrait conduire à une extrapolation des résultats relativement simple. Enfin le troisième contacteur sera un laveur de type jet de liquide pulvérisé dans un venturi pour absorber le gaz par dépression. Dans la pratique industrielle, ces trois réacteurs couvrent un domaine très large allant de l'oxydation du cyclohexane (première étape de la fabrication du nylon sel $\mathrm{N}$ ) pour le premier, à l'absorption de gaz nocifs comme le phosgène (pour le deuxième) et jusqu'à la captation de brouillards ou l'épuration de gaz de hauts fourneaux avec retenue éventuelle de particules solides pour le troisième.

Il sera montré à chaque fois comment les considérations sur les Sciences du Génie Chimique que j'ai présentées dans cette communication permettent de mieux appréhender la phénoménologie des transferts à l'échelle de l'interface gaz-liquide en vue d'une extrapolation ou d'une simulation de l'échelle industrielle. J'espère que l'esprit et la démarche pourront apporter une aide à ceux qui doivent choisir ou concevoir un réacteur gazliquide.

\section{Bibliographie}

[1] Réacteurs gaz-liquide et absorbeurs. Cycle de perfectionnement pour ingénieurs, Centre de Perfectionnement des Industries Chimiques, ENSIC, 1 rue Grandville, 54042 Nancy (France)

[2] CHARPENTIER J.C. - La Houille Blanche, 315, 5/1978.

[3] LAURENT A. - Thèse, Université de Nancy, I.N.P.L., 1975.

[4] CHARPENTIER J.C. - Pollution Atmosphérique, 66, 109 , 1975.

[5] CHARPENTIER J.C. - A review of the data on mass transfer in most of gas-liquid reactors, Edited by Kakac and Veziroglu, Hemisphere Publishing Corporation, 1977, pp. 869-910.

[6] CHARPENTIER J.C. - Gas-liquid reactors, Edited by Dan Luss and Weekman, American Chemical Symposium Series, 72, pp. 223-261, 1978.

[7] LAURENT A., PROST C., CHARPENTIER J.C. -- Joumal de Chimie Physique, 72, 236, 1975.

[8] LAURENT A., CHARPENTIER J.C. - Journal de Chimie Physique, 74, 1001, 1977.

[9] CHARPENTIER J.C. - Gas absorption and related separations, Advances in Chemical Engineering, Edited by Drew and Vermeulen, Academic Press, to be published in Volume $11(1980)$.

[10] ALPER E., DANCKWERTS P.V. - Chem. Eng. Sci., 31, $599,1976$.

[11] FONTEIX C. - Thèse, Université de Nancy, INPL, 1978. 